Conclusions The spleenic $\mathrm{CD} 4{ }^{+} \mathrm{CD} 25^{+}$treg and serum concentration of TGF- $\beta 1$, IL-10 of mice were decreased and there were lower frequency of Foxp $3^{+}$and $\mathrm{CD} 25^{+}$cells in severe atherosclerotic plaques than in mild. It meant that $\mathrm{CD} 4{ }^{+} \mathrm{CD} 25^{+}$treg may has antiatherosclerotic role on atherosclerotic progression.

\section{e0220 THE INVOLVEMENT OF IL-23/TH17 PATHWAY IN MURIN MODEL OF COXSACKIE VIRUS B3-INDUCED VIRAL MYOCARDITIS}

doi:10.1136/hrt.2010.208967.220

Fan Yang, Weifeng Wu, Yuluan Yan, Qing Kong, Yu Pang. Department of Cardiology, The First Affiliated Hospital of Guangxi Medical University, Nanning, China

Background The IL-23/Th17 pathway plays an important role in the development of chronic inflammatory diseases and autoimmune diseases. However, the role of the IL-23/Th17 axis in the regulation of virus myocarditis (VMC) is still largely unknown.

Methods VMC was induced in male $\mathrm{Balb} / \mathrm{c}$ mice by CVB3 peritoneal injection. Mice injected with PBS were taken as the controls. IL-23, IL-17 and ROR $\gamma \mathrm{t}$ mRNA in the myocardium of VMC were assessed by semi-quantitative RT-PCR on the time of $0,1,2,3,4$ and 6 weeks after injection. IL-23, IL-17 protein from blood plasma was evaluated by ELISA. Flow cytometric analysis was used to evaluate the frequencies of Th17 subsets in $\mathrm{CD}^{+}{ }^{+}$Tcell. CD4+ $\mathrm{T}$ cells were isolated from VMC mice and cultured with rIL-23 in vitro to investigate the function of IL-23 in the IL-23/Th-17 pathway.

Results Comparing with the controls, IL-23, IL-17 and ROR $\gamma$ t mRNA were steadily expressed in the myocardium of infected mice from 1 week after virus injection $(\mathrm{p}<0.01)$, IL-23 and IL17 protein level increased from 1 st week to 6 th week. The frequencies of Th17 cells were obviously increased in VMC mice 1 week after infection $(p<0.01)$, the maximum level of Th17 cells was reached at 4th week. The ratio of Th17 cells in the spleen lymphocyte significantly improved after rIL-23 stimulation, the IL-17 and ROR $\gamma \mathrm{t}$ mRNA expression of the cultured cells and the IL-17 protein in the culture supernatants increased after rIL-23 stimulation $(\mathrm{p}<0.05)$.

Conclusions IL-23/Th-17 pathway may play an essential role in VMC

\section{e0221 EFFECTS OF EXTRACORPOREAL CARDIAC SHOCK WAVE THERAPY ON EXPRESSION OF ENDOTHELIAL NITRIC OXIDE SYNTHASE AND BASIC FIBROBLAST GROWTH FACTOR IN SWINE WITH ACUTE MYOCARDIAL INFARCTION}

doi:10.1136/hrt.2010.208967.221

${ }^{1}$ Shuai Sun, ${ }^{2}$ Tao Guo. ${ }^{1}$ Department of Cardiology, First People's Hospital of Yunnan Province, Kunming, Yunnan, China; ${ }^{2}$ Department of Cardiology, First Affiliated Hospital of Kunming Medical College, Kunming, Yunnan, China

Objective To observe the effects of extracorporeal cardiac shock wave therapy (CSWT) on cintent of endothelial nitric oxide synthase (eNOS) and basic fibroblast growth factor (bFGF) in serum and myocardial tissue in swine with acute myocardial infarction (AMI).

Methods 12 Model swines with acute myocardial infarction was made, and were randomly divided into two groups with six in each group: simplex myocardial infarction as the control group and the experimental group which received CSWT treatment. (three times on the first, third, fifth days after operation. 200 hit/point. Total: 12 points. Energy: $0.09 \mathrm{~mJ} / \mathrm{mm}^{2}$ ). Peripheral blood was extracted at eight different time points before and after operation (immediate, the first, third, fifth days, 1, 2, 3, 4 weeks after operation) to detect serum eNOS content with enzymelinked immunosorbent assay.
Materials at myocardial tissue from the swines killed 1 month later were obtained to detect expression amount of eNOS and bFGF with semi quantitative RT-PCR method.

Results eNOS rose up in the experimental group 1 day after CSWT and a reached the peak on the fifth day, whereas eNOS gradully reduced in the control group, which slowed significant difference between the two groups $(p<0.01)$. Detection with semi quantities RT-PCR of myocardial tissues of infarction border area showed that eNOS and bFGF expression in the experimental group were obviously higher than those in the control group ((eNOS 27.705 \pm 4.13 ) vs $(16.448 \pm 3.21)$ (bFGF $32.571 \pm 4.23)$ vs $(17.858 \pm 4.17 \mathrm{p}<0.01))$.

Conclusions Extracorporeal cardiac shock wave therapy of acute myocardial infarction can effectively promote rise of eNOS and bFGF, which may be a new way to cure AMI.

\section{e0222 EFFECTS AND FUNCTION MECHANISM OF HYDROGEN SULFIDE ON MYOCARDIAL ISCHAEMIA REPERFUSION ARRHYTHMIA IN RATS}

doi:10.1136/hrt.2010.208967.222

${ }^{1}$ Chen Xiehui, 'Li Peng, ${ }^{1}$ Huang Xiaoping, ${ }^{2}$ Wang Dongming, ${ }^{2}$ Chen Songming, ${ }^{1}$ Yang Zeming, ${ }^{1}$ Chen Zhifan, ${ }^{1} X u$ Wenmin, ${ }^{1}$ Chen Liping. ${ }^{1}$ The 2nd People's Hospital of Futian District, Shenzhen, Guangdong, China; ${ }^{2}$ The First Affiliated Hospital of Shantou University Medical College, Shantou, Guangdong, China

Objective To explore the Effects and Function Mechanism of hydrogen sulfide on Myocardial Ischaemia reperfusion Arrhythmia in Rats.

Methods We used sodium hydrosulfide (NaHS) as the donor of $\mathrm{H}_{2} \mathrm{~S}$, $\mathrm{SD}$ rats were randomly divided into sham group, Myocardial Ischaemia reperfusion group (IR group), IR $+\mathrm{NaHS}$ group, and IR + $\mathrm{NaHS}+$ glibenclamide group. We monitor the Haemodynamics of rats, including heart rate, arterial pressure, left ventricular pressure et al. We also observe the rate of ventrical arrhythmia in each group. Result $\mathrm{H}_{2} \mathrm{~S}$ can significantly reduces rats' heart rate, arterial pressure and left ventricular pressure. It also reduces the rate of ventrical arrhythmia in Myocardial Ischaemia reperfusion Rats. The $\mathrm{K}_{\text {ATP }}$ Channel Blocker glibenclamide can weaken the $\mathrm{H}_{2} \mathrm{~S}^{\prime}$ Antiarrhythmic effects $(\mathrm{p}<0.01)$.

Conclusions $\mathrm{H}_{2} \mathrm{~S}$ can reduces the rate of ventrical arrhythmia in Myocardial Ischaemia reperfusion Rats. The Function Mechanism may be associated with the $\mathrm{K}_{\text {ATP }}$ signal transduction pathway in cells.

\section{e0223 EFFECTS OF NEOTYPE PERITONEAL COOLING ON THE INJURED OF INTESTINAL MUCOUS AFTER CARDIOPULMONARY RESUSCITATION IN RABBITS}

doi:10.1136/hrt.2010.208967.223

Xuan Dai, Xiao-xing Liao, Chun-Lin Hu, Hong-Yan Wei, Xin Li, Hong Zhan. Department of Emergency Medicine, The First Affiliated Hospital of Sun Yat-Sen University, Guangzhou, China

Objective To explore whether the peritoneal cooling after cardiopulmonary resuscitation could improve the injured of intestinal mucous in rabbits.

Methods 36 adult New Zealand rabbits were induced ventricular fibrillation by $\mathrm{AC}$ current. After the restore of spontaneous circulation (ROSC), rabbits were randomly divided into three groups according to the way of body temperature controlling, that is nomothermia group (NT), surface cooling group (SC) and peritoneal cooling group (PC). The changing of tympanic temperature and peritoneal temperature were observed after ROSC. The animals were sacrificed by over anaesthesia after ROSC for $12 \mathrm{~h}$, the end ileum was removed and fixed in formalin, the histological injured 
and the expression of TNF-a and VCAM-1 in ileum were observed by $\mathrm{H}$.E staining and immune chemical methods

Results 12 animals in each group, 9 in group NT, 10 in group SC and 9 in group PC were successfully resuscitated; all animals were on mechanical ventilation for 2 to $4 \mathrm{~h} \mathrm{5,6}$ and 8 animals in each group respectively survived to the end of the experiment. The temperatures of tympanic and peritoneal cavity of animals in group NT were maintained in normal range. The tympanic temperature of animals in group SC and PC was arrived target temperatures at $29 \pm 6.55$ mins and $62 \pm 8.27$ mins. During the stage of maintenance of hypothermia, the tympanic and peritoneal temperatures of animals in group SC were in range 33 to $35^{\circ} \mathrm{C}$, while the peritoneal temperatures of animals in group $\mathrm{PC}$ were in range 31 to $34^{\circ} \mathrm{C}, 1$ to $2^{\circ} \mathrm{C}$ lower than the tympanic temperature. The scores of histological injured of ileum were $1.43 \pm 0.53$ in group PC, $3.4 \pm 0.55$ in group NT and $3.17 \pm 0.41$ in group SC. The differences among them were significantly, PC vs SC, $\mathrm{p}<0.000$; PC vs NT, $\mathrm{p}<0.000$; while SC vs NT, $\mathrm{p}=0.30$. The expression of TNF-a in ileum was $9.98 \pm 1.79 \%$ in group NT, $5.87 \pm 1.43 \%$ in group SC and $3.78 \pm 0.53 \%$ in group PC, the differences among them were significantly. The phenomenon of the expression of VCAM-1 was little like the TNF-a, $3.78 \pm 0.53 \%$ in group PC was significantly from the $8.53 \pm 1.53 \%$ in group NT and $5.92 \pm 1.06 \%$ in group SC.

Conclusion The neotype peritoneal cooling can improve the injured of ileum mucous beside quickly induce hypothermia after ROSC in rabbits.

\section{e0224 MODEL OF CARDIAC ARREST IN RATS BY TRANSCUTANEOUS ELECTRICAL EPICARDIUM STIMULATION}

doi:10.1136/hrt.2010.208967.224

\begin{abstract}
${ }^{1}$ Lin Ji-yan, ' ${ }^{1}$ Li Xin, ${ }^{1}$ Li Hui, ' Wei Hong-yan, 'Liu Rong, ${ }^{1} H u$ Chun-lin, ${ }^{2}$ Dai Gang, ${ }^{1}$ Liao Xiao-xing. ${ }^{1}$ Department of Emergency Medicine, The First Affiliated Hospital of Sun Yat-Sen University, Guangdong, China; ${ }^{2}$ Key Lab on Assisted Circulation of Ministry of Health of Sun Yat-Sen University, Guangzhou, China
\end{abstract}

Objective To establish a new model of Cardiac Arrest (CA) in rats by transcutaneous electrical epicardium stimulation.

Methods Two acupuncture needles connected to the anode and cathode of a stimulator were transcutaneously inserted into the epicardium as electrodes. The stimulating current was steered to the epicardium and the stimulation was maintained for 3 min to induce CA. Cardiopulmonary resuscitation (CPR) was performed at 6 min after a period of nonintervention.

Results The success rate of induction was $12 / 20$ at the current intensity of $1 \mathrm{~mA}$; and reached 20/20 when the current intensity was increased to $2 \mathrm{~mA}$. The average time from the electrical stimulation to CA induction was $5.10( \pm 2.81) \mathrm{s}$. When the electrical stimulation stopped, 18/20 rats had ventricular fibrillation and $2 / 20$ rats had pulseless electrical activity. CPR was performed for averagely $207.4( \pm 148.8) \mathrm{s}$. The restoration of spontaneous circulation was $20 / 20$. The death rate within $4 \mathrm{~h}$ after CA was $5 / 20$, and the $72-$ h survival rate was $10 / 20$. There were only two cases of complications, a minor muscle contraction and a minor lung lobe injury.

Conclusion The model of CA in rats induced by transcutaneous electrical epicardium stimulation is a stable model that requires lowintensity current and has fewer complications.

\section{e0225 HYDROGEN SULFIDE INHABITS NEURONS APOPTOSIS IN RATS AFTER CARDIOPULMONARY RESUSCITATION}

doi:10.1136/hrt.2010.208967.225

${ }^{1}$ Xiao-xing Liao, ${ }^{1} \mathrm{Ji}$-yan Lin, ${ }^{1} \mathrm{H}$ ong-yan Wei, ${ }^{1} \mathrm{Hui}$ Li, ${ }^{1} \mathrm{Xin} \mathrm{Li}$, ${ }^{1}$ Rong Liu, ${ }^{1}$ Chun-lin Hu, ${ }^{1}$ Guo-qing Huang, ${ }^{2}$ Gang Dai. ${ }^{1}$ Department of Emergency, the First Affiliated Hospital of
Sun Yat-Sen University, Guangzhou, China; ${ }^{2}$ Key Lab on Assisted Circulation of Ministry of Health of Sun Yat-Sen University, Guangzhou, China

Objective To investigate the effects of hydrogen sulfide $\left(\mathrm{H}_{2} \mathrm{~S}\right)$ on brain injury after cardiopulmonary resuscitation (CPR) in rats by examining neurons apoptosis.

Methods The 40 male SD rats were randomly divided into experimental and control groups equally. In control group, CPR was performed with Utstein mode at 6 min after CA. On this basis, sodium hydrosulfide was administrated to the rats after restoration of spontaneous circulation in experimental group. On seventh day after CPR, neurons apoptosis was examined using terminal deoxynucleotidyl transferase mediated dUTP biotin nick end labelling (TUNEL) staining and the expression of caspase-3 was detected by the immunohistochemical strepto avidin biotinperoxidase complex (SABC) method in cortex, hippocampus CA1 region and cerebellum of the rats.

Results 1 . There were 12 and 10 rats completed the experiment in the experimental and control group respectively. Their fate between the two groups was no significant difference $\left(\chi^{2}=0.404, p=0.376\right) .2$. On seventh day after $\mathrm{CPR}$, The serum concentrations of $\mathrm{H}_{2} \mathrm{~S}$ was $9.12 \pm 3.17 \mu \mathrm{mol} / 1$ in the experimental group and the contrast was $3.72 \pm 1.05 \mu \mathrm{mol} / \mathrm{l}$, the difference between the two groups had statistic significance $(t=5.136, p=0.000)$. 3. Compared with the control group, the experimental group's neurons apoptosis index and the sum of integrated optical density (IOD) of caspase- 3 in cortex, hippocampus CA1 region and cerebellum were obviously reduced $(p<0.05)$.

Conclusion After CPR, $\mathrm{H}_{2} \mathrm{~S}$ can inhabit neurons apoptosis and its mechanism may be through caspase- 3 pathway. It may play a role in the treatment of the brain injury after CA.

\section{e0226 EFFECTS OF BONE MARROW MESENCHYMAL STEM CELLS ON ELECTROPHYSIOLOGICAL FUNCTION IN RATS WITH MYOCARDIAL INFARCTION}

doi:10.1136/hrt.2010.208967.226

Wang Deguo, Zhang Fengxiang, Shen Wenzhi, Chen Hongwu, Yang Bing, Chen Minglong, Cao Kejiang. The First Affiliated Hospital of Nanjing Medical University, Nanjing, Jiangsu, China

Objective Concerns that intramyocardial delivery of immature cells could cause potentially life-threatening ventricular arrhythmias have been repeatedly raised. The aim of this study is to investigate the electrophysiological and arrhythmogenic effects for MSCs therapy in AMI.

Methods GFP tagged MSCs were injected into a murine heart with left anterior descending (LAD) ligation. Two weeks after transplantation, effective refractory period (ERP), ventricular arrhythmias (VAs) inducibility and ventricular fibrillation threshold (VFT) were assessed by programmed electrical stimulation (PES), respectively. Epicardial monophasic action potential (MAP) recordings were obtained from infarcted border zone (IBZ) and none infarcted zone (NIZ) of left ventricular epicardium for calculation action potential duration (APD) and activation time (AT). Immunofluorescence and immunoblots were used to determine the expression and distribution of $\mathrm{Cx} 43$, collagen I and Kv4.2.

Results PES showed a significant reduced VTs, raised VFT and VERP in MSCs treated rats compared to PBS treated animals. MSCs implantation led to markedly longer APD and shorter AT in IBZ than PBS treated hearts. Histological study revealed that fibrotic area and collagen deposition in infarcted region were significantly lower in MI-MSCs group than in MI-PBS group. Abnormal alterations of $\mathrm{Cx} 43$ including reduction and lateralisation were significantly attenuated by MSCs treatment. Inhibition of Kv4.2 expression was partly ameliorated by MSCs therapy. 